\title{
GALANIN (1-15) ENHANCEMENT OF THE BEHAVIORAL EFFECTS OF FLUOXETINE IN THE FORCED SWIMMING TEST GIVES A NEW THERAPEUTIC STRATEGY AGAINST DEPRESSION.
}

Antonio Flores-Burgess $\mathrm{MsC}^{1}$, Carmelo Millón $\mathrm{PhD}^{1}$, Belen Gago $\mathrm{PhD}^{1}$, Manuel Narváez MD, PhD ${ }^{1}$, Dasiel O. Borroto-Escuela $\mathrm{PhD}^{2}$, Guadalupe Mengod $\mathrm{PhD}^{3}$, José Angel Narváez MD, $\mathrm{PhD}^{1}$, Kjell Fuxe MD, $\mathrm{PhD}^{2}$, Luis Santín $\mathrm{PhD}^{4}$ \& Zaida Díaz-Cabiale $\mathrm{PhD}^{1}$

1.Universidad de Málaga, Instituto de Investigación Biomédica de Málaga, Facultad de Medicina, Campus de Teatinos s/n, 29071 Málaga, Spain.

2. Department of Neuroscience, Karolinska Institute, Stockholm, Sweden.

3. Department of Neurochemistry and Neuropharmacology, IIBB-

CSIC,IDIBAPS,CIBERNED, Barcelona 08036, Spain.

4.Universidad de Málaga, Instituto de Investigación Biomédica de Málaga, Facultad de Psicología, Campus de Teatinos s/n, 29071 Málaga, Spain.

${ }^{*}$ Corresponding author:

Z. Díaz-Cabiale

Universidad de Málaga

Instituto de Investigación Biomédica de Málaga

Facultad de Medicina

Campus de Teatinos s/n, 29071 Málaga, Spain

Fax: + 34-952131650 Tel.: + 34-952134223

E-mail: zaida@uma.es

Running tittle: Galanin(1-15) enhances Fluoxetine effect 


\section{Abstract}

The pharmacological treatment of major depression is mainly based on drugs elevating serotonergic (5-HT) activity. Specifically, selective 5-HT reuptake inhibitors, including Fluoxetine (FLX), are the most commonly used for treatment of major depression. However, the understanding of the mechanism of action of FLX beyond its effect of elevating 5-HT is limited. The interaction between serotoninergic system and neuropeptides signalling could be a key aspect. We examined the ability of the neuropeptide Galanin(1-15) [GAL(1-15)] to modulate the behavioral effects of FLX in the forced swimming test (FST) and studied feasible molecular mechanisms. The data show that GAL(1-15) enhances the antidepressant-like effects induced by FLX in the FST, and we demonstrate the involvement of GALR1/GALR2 heteroreceptor complex in the GAL(1-15)-mediated effect using in vivo rat models for siRNA GALR1 or GALR2 knockdown.

Importantly, 5-HT1A receptors (5HT1AR) also participate in the GAL(1-15)/FLX interactions since the 5HT1AR antagonist WAY100635 blocked the behavioral effects in the FST induced by the coadministration of GAL(1-15) and FLX. The mechanism underlying $\mathrm{GAL}(1-15) / F L X$ interactions affected the binding characteristics as well as the mRNA levels of 5-HT1AR specifically in the dorsal hippocampus while leaving unaffected mRNA levels and affinity and binding sites of this receptor in the dorsal raphe. The results open up the possibility to use GAL(1-15) as for a combination therapy with FLX as a novel strategy for treatment of depression. 


\section{INTRODUCTION}

Major Depression is the most frequent mood disorder, with a lifetime prevalence that has been reported to range from $7 \%$ to $21 \%$ (Albert et al., 2011). It is associated with a substantial functional impairment, diminished quality of life, increased burden both for patients and caregivers, as well as with a higher risk of mortality.

Although several neurotransmitter systems and brain areas have been implicated in this pathology, the pharmacological treatment of major depression is mainly based on drugs elevating serotonergic (5-HT) activity (Blier and de Montigny, 1994; Jans et al., 2007). Specifically, selective 5-HT reuptake inhibitors (SRRIs) are the most commonly used for treatment of major depression. In particular, Fluoxetine (FLX) is usually chosen for the treatment of symptoms of depression (Artigas, 2013). However the understanding of the mechanism of action of FLX beyond its effect of elevating $5-\mathrm{HT}$ is limited. Recent studies have also implicated several neuropeptides in the pathophysiology of depression in rodents and in the possibility to use them as a combined treatment with antidepressant drugs.

In this context, the neuropeptide Galanin (GAL) (Tatemoto et al., 1983) is of particular interest. GAL is widely distributed in neurons within the Central Nervous System (CNS) and three GAL receptor (GALR) subtypes, GALR1-3 with a high affinity for GAL have been cloned (Branchek et al., 2000; Mitsukawa et al., 2008). GALR1 and GALR3 mainly activate inhibitory G proteins Gi/Go, while GALR2 mainly couples to $\mathrm{Gq} / \mathrm{G} 11$ to mediate excitatory signaling (Branchek et al., 2000; Wang et al., 1997).

GAL participates in mood regulation and depression in rodents (Bellido et al., 2002; Juhasz et al., 2014; Wang et al., 2016; Weiss et al., 1998). Thus, the activation of GALR1 and GALR3 results in a depression like behavior while stimulation of GALR2 leads to anti-depressant-like effects (Bartfai et al., 2004; Kuteeva et al., 2008; Lu et al., 2005). Recently, it has been described that GAL and its receptor GALR3 are differentially methylated and expressed in brains of major depression subjects in a region- and sex-specific manner (Barde et al., 2016). 
In this context, GAL modulates $5-\mathrm{HT} 1 \mathrm{~A}$ receptor $(5-\mathrm{HT} 1 \mathrm{AR})$ function at autoreceptor and postsynaptic level in the brain. In the dorsal raphe (DR), intracerebroventricular (icv) GAL induced a time-dependent reduction in affinity and an increase in the 5-HT1AR autoreceptor density( Razani et al., 2001). At the post-synaptic level, GAL reduced the affinity of the 5-HT1AR in the ventral limbic cortex (Fuxe et al., 1988; Hedlund and Fuxe, 1996). Moreover, in hypothermia, locomotor activity and passive avoidance, icv GAL blocked post-synaptic 5-HT1AR function (Kehr et al., 2002; Misane et al., 1998; Razani et al., 2001). The antagonistic GALR-5-HT1AR interactions described in the brain, especially in the limbic forebrain regions and in the dorsal raphe, has been suggested to be of high relevance for depression (Fuxe et al., 1988; Hedlund and Fuxe, 1996; Kehr et al., 2002; Misane et al., 1998; Razani et al., 2001).

Not only GAL but also the N-terminal fragments like GAL(1-15) are active in the CNS (Diaz-Cabiale et al., 2007; Diaz-Cabiale et al., 2010; Diaz-Cabiale et al., 2005; Hedlund and Fuxe, 1996). Recently we described that GAL(1-15) induces strong depression-related and anxiogenic-like effects in rats and these effects were significantly stronger than the ones induced by GAL (Millon et al., 2015). The GALR1-GALR2 heteroreceptor complexes in the dorsal hippocampus and $D R$, areas rich in GAL(1-15) binding sites (Hedlund et al., 1992) were involved in these effects (Millon et al., 2015) and demonstrated also in cellular models (Borroto-Escuela et al., 2014).

GAL(1-15) is also able to enhance the antidepressant effects induced by the 5HT1AR agonist 8-OH-DPAT in the forced swimming test (FST) (Millon et al., 2016), effect that was again significantly stronger than the ones induced by GAL. Importantly, the mechanism underlying this action involved interactions at the receptor level in the plasma membrane with changes also at the transcriptional level. Thus, GAL(1-15) affected the binding characteristics as well as the mRNA levels of 5-HT1AR in the dorsal hippocampus and DR (Millon et al., 2016). 
In view of these results the purpose of the current study was to assess the ability of GAL(1-15) to modulate the behavioral effects of FLX. We have analyzed the effect of GAL (1-15) on FLX-mediated responses in a behavioral test of depression. We tested the involvement of GALR2 in the GAL (1-15) effect with the selective GALR2 antagonist M871 and using an in vivo rat model for siRNA GALR2 knockdown. The role of the GALR1 in the behavioral actions of GAL(1-15) were analyzed using an in vivo rat model for siRNA GALR1 knockdown. Moreover, to study wether the effects of $\mathrm{GAL}(1-15)$ on FLX action were mediated via 5-HT1AR, we have analyzed the effect of the 5-HT1AR antagonist WAY100635 in the GAL(1-15)-mediated effect in the FST. We have also determined the binding characteristics and mRNA levels of 5-HT1AR in the DR and dorsal hippocampus after GAL(1-15)-FLX administration.

\section{MATERIALS AND METHODS}

\section{Animals}

Male Sprague-Dawley rats obtained from CRIFFA, Barcelona (200-250gr) were maintained under a $12 \mathrm{~h}$ dark/light cycle in temperature- and humidity-controlled conditions $\left(22 \pm 2^{\circ} \mathrm{C}, 55-60 \%\right)$. The animals had free access to food pellets and tap water. Behavioural tests were performed during the light phase of the diurnal cycle. All Experimental procedures were approved by the Institutional Animal Ethics Committee of the University of Málaga, Spain.

Detailed descriptions on animal controlled-conditions, surgical preparation, icv injections, forced swim test (FST) and behavioral scores are available in Supplemental Information.

\section{Forced Swimming Test}

Previously to the test, the animals were adapted to experimental room and handled. All tests were performed between 13:00h and 16:00h during the light cycle. Two swimming sessions were conducted: a 15 min pre-test followed $24 \mathrm{~h}$ later by a 5 min test. Animals were individually placed in a vertical glass cylinder of $20 \mathrm{~cm}$ diameter containing water $\left(25^{\circ} \mathrm{C}\right)$ to a height of $30 \mathrm{~cm}$. (Detke and Lucki 1996; Kuteeva et al. 2007). The total duration of immobility, climbing and 
swimming behavior were recorded during the second $5 \mathrm{~min}$ test. The administration of drugs was performed between sessions.

siRNA GALR1 and GALR2 Knockdown Rats.

Knockdown rats were generated as previously described (Millon et al., 2015). Using real time quantitative PCR, we have previously performed a time course of GALR2 mRNA in dorsal hippocampus (Millon et al., 2015) and we had also performed a time course of GALR2 protein expression using quantification of immunohistochemical staining for GALR2 in hippocampus (Millon et al., 2015). The time course curve indicated a maximal reduction of GALR2 receptor protein expression 8 days after the injection (Millon et al., 2015).

Briefly, during the stereotaxic surgery, once the cannula is fixed, animals received an intracerebroventricular (icv) injection of $5 \mu \mathrm{g}(0.35 \mathrm{nmol})$ of Accell Smart pool siRNA for GALR2 or GALR1 (Dharmacon). Animals had a recovery period of 8 days prior to behavioural test, the time required to reduce the levels of GAL receptors as previously described (Millon et al., 2015).

\section{Behavioural Assessment}

Groups of rats were assessed in the FST. The doses, injection schedule and mode of administration of substances were chosen based on previous work (Estrada-Camarena et al., 2003; Estrada-Camarena et al., 2006; Jorgensen et al., 2001; Millon et al., 2016).

In the first set of experiments, we performed a dose-response curve of FLX. Groups of rats received three injections subcutaneously (sc) of FLX at the doses of $10 \mathrm{mg} / \mathrm{Kg}$ or $2.5 \mathrm{mg} / \mathrm{Kg}$, or vehicle 23,5 and 1 hour before the tests and an icv injection of artificial cerebrospinal fluid (aCSF) 15 min before the test.

This pattern of injection was shown to produce effects of FLX in the FST similar to those obtained after chronic treatment and to those leading to its clinical effects in humans (Detke et al., 1997). This mode of injection was used in all the experiments of this study.

In the second set of experiments, in order to evaluate the interaction of FLX with $\mathrm{GAL}(1-15)$ in the FST, groups of rats received three injections of $\mathrm{sC}$ 
FLX $(2.5 \mathrm{mg} / \mathrm{Kg})$ or $F L X(10 \mathrm{mg} / \mathrm{Kg})$ and a single icv injection of a threshold dose of GAL(1-15)(1nmol) 15 minutes before the test. Also we compared the effects between the coadministration of $\mathrm{FLX}(10 \mathrm{mg} / \mathrm{Kg})$ and $\mathrm{GAL}(1-15)(1 \mathrm{nmol})$ or GAL(1 $1 \mathrm{nmol})$ in the FST.

In a third set of experiments we determined the involvement of GALR1 and GALR2 in the effect of GAL(1-15) on FLX-mediated action. Groups of rats received three injections of sc FLX $(10 \mathrm{mg} / \mathrm{kg})$, a single icv injection of $\mathrm{GAL}(1-15)$ (1 $\mathrm{nmol})$ and the GALR2 antagonist M871 (3nmol) icv alone or in combination. Also, in siRNA GALR1 or GALR2 knockdown rats we coadministered $\mathrm{FLX}(10 \mathrm{mg} / \mathrm{Kg})$ and $\mathrm{GAL}(1-15)(1 \mathrm{nmol})$ in order to analyze the involvement of GALR1 and GALR2 receptors in the GAL(1-15)-mediated effect.

To further study if the effects of GAL(1-15) on FLX action were mediated via the $5 \mathrm{HT} 1 \mathrm{AR}$, groups of rats received three injections of sc FLX $(10 \mathrm{mg} / \mathrm{kg})$ and 15 minutes before the test a single icv injection of $\operatorname{GAL}(1-15)(1 \mathrm{nmol})$ and 5 HT1AR antagonist WAY100635(6nmol) icv alone or in combination.

\section{Autoradiography and in situ hybridization histochemistry}

The procedure to perform receptor autoradiography and in situ hybridization histochemistry was previously described (Millon et al., 2015; Saenz del Burgo et al., 2013). Rats ( $n=6$ per group) received the three injections of sc FLX or Vehicle and a single icv injection of GAL(1-15) or aCSF, and were sacrified 30 min later. Coronal sections were obtained at the dorsal hippocampus and DR level (see Supplemental Information for details).

\section{Quantitative autoradiography}

Saturation experiments were performed using $\left[{ }^{3} \mathrm{H}\right]-8-\mathrm{OH}-D P A T$. Briefly, the sections were preincubated for 30 minutes at room temperature in $50 \mathrm{mM}$ Tris$\mathrm{HCl}$ buffer $(\mathrm{pH} 7.6)$ containing $4 \mathrm{mM} \mathrm{CaCl}_{2}, 0.01 \%$ ascorbic acid and $10 \mathrm{mM}$ pargyline. The sections were then incubated for 60 minutes at room temperature with $\left[{ }^{3} \mathrm{H}\right]-8-\mathrm{OH}-\mathrm{DPAT}$ in the same solution as above. Film exposure time for sections was 6 weeks (see Supplemental Information for details). 
In situ hybridization histochemistry

The oligonucleotides complementary to the mRNA coding for 5-HT1AR are found in Supplemental information. Oligonucleotide labeling, in situ hybridization histochemistry and specificity controls have been described elsewhere (Pompeiano et al., 1992; Tomiyama et al., 1997).

Hybridized sections were exposed to Kodak Biomax-MR (Sigma Aldrich) for 10 days.

Image analysis

Measurements were made in the Dentate Gyrus (DG) and the CA1 area of the hippocampus $\left(0.15 \mathrm{~mm}^{2}\right)$. The ventral part of the midline area of the DR was analyzed using a square as a sampling field $\left(0.09 \mathrm{~mm}^{2}\right)$ (see Supplemental Information for details).

\section{Statistical Analysis:}

Data are presented as the mean \pm SEM and samples number (n) are indicated in figure legends. All data were analyzed using GraphPad PRISM 4.0 (GraphPad Software, La Jolla, CA). For comparing two experimental conditions, Student's unpaired t-test was performed. Otherwise, one-way analysis of variance (ANOVA) followed by Newman-Keuls comparison post hoc-test was performed. Differences were considered significant at $\mathrm{P}<0.05 \quad\left({ }^{*} \mathrm{P}<0.05, \quad{ }^{* *} \mathrm{P}<0.01\right.$, $\left.{ }^{* \star *} P<0.001\right)$. The data from the saturation experiments were analyzed by nonlinear regression analysis for the determination of the dissociation constant $(\mathrm{Kd})$ and the total number of agonist binding sites (Bmax) using GraphPad PRISM 4.0.

\section{RESULTS}

\section{Behavioral effects}

Dose-response to Fluoxetine

As previously described, three injections of FLX given sc at $10 \mathrm{mg} / \mathrm{Kg}$ induced an antidepressant-like effect in the FST in rats (Table S1). The overall one way 
ANOVA revealed a significant effect between treatments in immobility time $\left(F_{2,20}=4.628, p<0.05\right)$ and swimming time $\left(F_{2,20}=7.044, p<0.01\right)$ while no significant difference was observed in climbing time $\left(F_{2,20}=2.569, p=0.102\right)$. $\mathrm{FLX}(10 \mathrm{mg} / \mathrm{Kg}$ ) reduced the immobility (post hoc, $\mathrm{p}<0.05)$ and increased swimming time (post hoc, $p<0.01$ ) compared to controls and FLX $2.5 \mathrm{mg} / \mathrm{Kg}$. The three injections of sc FLX $2.5 \mathrm{mg} / \mathrm{Kg}$ was considered as threshold in this test since it did not produce antidepressant-like effects compared to controls (Table S1).

GAL(1-15) but not GAL enhances antidepressant-like effects of FLX in the FST

The coadministration of the three sc injections of the threshold dose of FLX $(2.5 \mathrm{mg} / \mathrm{Kg})$ and a single icv injection of $\mathrm{GAL}(1-15)(1 \mathrm{nmol})$ (Figure S1) induced antidepressant-like effects with a significant decrease in the immobility $\left(F_{3,27}=3.973, p<0.05\right)$ compared with the rest of the groups (Figure $S 1$; post hoc $p<0.05)$. Moreover, an increase in the swimming time $\left(F_{3,28}=4.137, p<0.05\right)$ compared with GAL(1-15) and controls was also observed (Figure S1; post hoc, $\mathrm{p}<0.05)$.

The strong enhancement by $\mathrm{GAL}(1-15)$ of the antidepressant-like effects mediated by FLX was validated using the effective dose of FLX $10 \mathrm{mg} / \mathrm{kg}$ in the FST. The threshold dose of GAL(1-15) $1 \mathrm{nmol}$ enhanced the antidepressant-like effects mediated by the three sc injections of $F L X(10 \mathrm{mg} / \mathrm{kg})$ since the overall one way ANOVA revealed a reduction in the immobility time $\left(F_{3,28}=10.96\right.$, $\mathrm{p}<0.001)$ and an increase in the swimming time $\left(\mathrm{F}_{3,28}=16.46, \mathrm{p}<0.001\right)$ without effects on climbing time $\left(\mathrm{F}_{3,27}=0.7554, \mathrm{p}=0.5289\right)$ (Fig. 1). Icv $\mathrm{GAL}(1-15)$ significantly decreased the immobility time induced by the three injections of the effective dose of FLX $(10 \mathrm{mg} / \mathrm{kg})$ by $50 \%$ in the FST (Figure 1; post hoc $p<0.05$ ). Moreover, an increase of the swimming time by about $40 \%$ versus FLX $(10 \mathrm{mg} / \mathrm{kg})$ group was also observed $(p<0.01)$.

The coadministration of three sc injections of $F L X(10 \mathrm{mg} / \mathrm{Kg})$ and a single icv injection of GAL(1nmol) lacked effects on the immobility and swimming behaviours compared to FLX $(10 \mathrm{mg} / \mathrm{kg})$ in the FST (Figure 1). 
GAL Receptor subtypes involved in the GAL(1-15)-Fluoxetine interaction

We tested the involvement of the GALR2 in the GAL(1-15)-FLX $(10 \mathrm{mg} / \mathrm{kg})$ interaction with the selective GALR2 antagonist M871 and using an in vivo rat model of siRNA GALR2 knockdown.

\section{Effects of the GALR2 antagonist M871}

The GALR2 antagonist M871 3nmol significantly blocked the GAL(1-15)induced reduction of the immobility time $\left(F_{3,28}=10.20, p<0.001\right.$; post hoc, $p<0.05)$, and the $G A L(1-15)$-induced increase in the swimming time $\left(F_{3,29}=15.75, p<0.001 ; p o s t\right.$ hoc, $\left.p<0.01\right)$ found after coadministration of a single icv injection of $\operatorname{GAL}(1-15)$ and the three sc injections of $F L X(10 \mathrm{mg} / \mathrm{kg})$ in the FST (Figure 2A). The GALR2 antagonist M871 injected alone in the dose of 3 nmol lacked effects on immobility time $(141 \pm 12 \mathrm{sec})$ and swimming time $(68.7 \pm 10 \mathrm{sec})$ in the FST.

\section{Effects of siRNA GALR2 knockdown}

Then, we used knockdown GALR2 rats to establish the involvement of the GALR2 in the interaction between GAL $(1-15)(1 \mathrm{nmol})$ and $\mathrm{FLX}(10 \mathrm{mg} / \mathrm{kg})$ (Figure 2B).

The one-way ANOVA revealed a significant effects of the treatment used in the immobility $\left(\mathrm{F}_{2,17}=5.932, \mathrm{p}<0.05\right)$ and swimming behaviours $\left(\mathrm{F}_{2,17}=5.932\right.$, $p<0.01)$. The three sc injections of $F L X(10 \mathrm{mg} / \mathrm{kg})$ produced antidepressant-like effects in the siRNA GALR2 knockdown animals with a decrease in the immobility time (Figure $2 \mathrm{~B}$; post hoc $\mathrm{p}<0.05$ ) and an increase in the swimming time (Figure $2 \mathrm{~B}$; post hoc $\mathrm{p}<0.01$ ) compared to a control group of siRNA GALR2 knockdown rats. Moreover, the coadministration of the three sc injections of $F L X(10 \mathrm{mg} / \mathrm{kg})$ and a single icv injection of $\mathrm{GAL}(1-15)$ in siRNA GALR2 knockdown animals did not produce a further reduction of the immobility time and a further increase in the swimming time compared to FLX alone (Figure 2B). GAL(1-15)(1nmol) injected alone lacked effects in the FST in knockdown GALR2 animals compared to control group in terms of immobility (14.7 $\pm 3 \mathrm{~s})$, swimming ( $81.7 \pm 3 \mathrm{~s})$ and climbing times $(60 \pm 4 \mathrm{~s})$. 


\section{Effects of siRNA GALR1 knockdown}

An siRNA GALR1 knockdown in rats was used to determine the involvement of the GALR1 in the effect induced by GAL(1-15) on FLX in the FST. In this model, previously used in our laboratory, it was possible to validate a reduction by $30 \%$ of the expression of GALR1 by immunochemical assay in the CA1 area of the dorsal hippocampus (Figure $3 C ; \mathrm{t}_{14}=2.317, \mathrm{p}<0.05$ ) and DG (Figure 3C; $\left.\mathrm{t}_{14}=2.359, \mathrm{p}<0.05\right)$.

In the FST on the siRNA GALR1 knockdown rats, the three sc injections of $\mathrm{FLX}(10 \mathrm{mg} / \mathrm{Kg})$ produced antidepressant-like effects with a decrease in the immobility time (Figure $3 A ; t_{16}=2,778, p<0,05$ ) and an increase in the swimming time (Figure $3 A ; t_{16}=2,041, p<0.05$ ) compared to the control group of GALR1 knockdown rats. Moreover, in a different set of experiments, the coadministration of the three sc injections of $F L X(10 \mathrm{mg} / \mathrm{Kg})$ and a single icv injection of GAL(1-15)(1nmol) in GALR1 knockdown animals did not produce a statistically significant difference compared to $F L X(10 \mathrm{mg} / \mathrm{kg})$ group alone in the immobility time (Figure $3 \mathrm{~B} ; \mathrm{t}_{15}=0.121$ ) nor in the swimming time (Figure 3B; $\left.\mathrm{t}_{14}=0.319\right)$.

\section{$5 H T 1 A R$ is involved in the GAL (1-15)-FLX interaction}

To determine the role of $5 \mathrm{HT} 1 \mathrm{AR}$ in the behavioral interaction between GAL(115) and FLX the 5HT1AR antagonist WAY100635 was used (Figure 4).

The results confirmed that the 5HT1AR participates in this interaction as the 5HT1AR antagonist WAY100635 (6nmol) significantly blocked the reduction in immobility time $\left(F_{3,30}=4.243, p<0.05\right.$;post hoc, $\left.p<0.05\right)$, and the increase in swimming time $\left(\mathrm{F}_{3,29}=16.83, \mathrm{p}<0.001\right.$;post hoc, $\left.\mathrm{p}<0.01\right)$ induced by the coadministration of icv GAL(1-15) and the three injections of sc FLX $(10 \mathrm{mg} / \mathrm{kg})$ in the FST (Figure 4).

The 5HT1AR antagonist WAY100635 (6nmol) injected alone significantly increased the immobility time $(101 \pm 12 \mathrm{sec})\left(\mathrm{t}_{13}=2,617, \mathrm{p}<0,01\right)$ and lacked effect in swimming time $(87 \pm 6 \mathrm{sec})\left(\mathrm{t}_{13}=0,2135, \mathrm{p}=0.4172\right)$ in the FST. However, the coadministration of three sc injections of FLX and a single icv 
injection of WAY100635 $(6 \mathrm{nmol})$ did not produce any difference compared with the effect of FLX alone (Figure 4).

\section{Neurochemical effects}

GAL(1-15)+FLX modify the mRNA levels and the binding characteristics of 5HT1AR in the dorsal hippocampus.

\section{5-HT1A mRNA levels}

The coadministration of the three sc injections of $F L X(10 \mathrm{mg} / \mathrm{Kg})$ and a single icv injection of GAL(1-15)(1nmol) produced a significant increase in the $5 \mathrm{HT} 1 \mathrm{AR}$ mRNA levels in CA1 (Figure 5; $F_{3,18}=8.803, p<0.001$; post hoc, $p<0.05$ ) and $D G$ (Figure 4; $F_{3,17}=5.033, p<0.05$; post hoc, $p<0.05$ ) of the dorsal hippocampus compared to controls and single treatments. This effect was not observed in the DR (Table S2; $F_{3,19=0.983 ; ~}=0.422$ ).

Representative autoradiograms illustrate the increase of 5HT1AR mRNA levels in both areas, $C A 1$ and $D G$, after the coadministration of the three sc injections of FLX (10mg/Kg) and the single icv injection of GAL(1-15)(1nmol) (Figure $5 \mathrm{~B})$.

\section{5-HT1A agonist radioligand binding. Saturation curves}

In the autoradiographic experiments, the coadministration of the three injections of sc FLX $(10 \mathrm{mg} / \mathrm{Kg})$ and a single icv injection of GAL(1-15)(1nmol) produced a significant decrease in the $\mathrm{Kd}$ value (Figure 6; $\mathrm{t}=2.916, \mathrm{p}<0.01$ ) and in the Bmax value (Figure 6 ; $t_{9}=2.277, p<0.05$ ) of the agonist radioligand $\left[{ }^{3} \mathrm{H}\right]-8-\mathrm{OH}$ DPAT in the DG of the dorsal Hippocampus compared to $F L X(10 \mathrm{mg} / \mathrm{Kg})$ alone. Representative autoradiograms with a low radioligand concentration (1nM) illustrate the increase of labelling (decrease in Kd values) in the DG 30 minutes after the coadministration of the three injections of sc FLX $(10 \mathrm{mg} / \mathrm{Kg})$ and the single icv injection of GAL1-15 (1nmol) (Figure 6B).

These effects were not observed in the CA1 area of the Hippocampus neither in the Bmax (Table S3; $t_{9}=0,9420, p=0,1854$ ) nor in the $K d$ values (Table S3; $t_{9}=0,01385, p=0,4946$ ) neither in the DR in the Bmax (Table S3; $t_{9}=0,8648$, 
$\mathrm{p}=0,2048)$ or in the Kd (Table S3; $\left.\mathrm{t}_{9}=0,9941, \mathrm{p}=0,173\right)$.

\section{DISCUSSION}

In the current study we describe for the first time that $G A L(1-15)$ enhances the antidepressant-like effects induced by FLX in the FST. These effects were specific since GAL lacks effect. Indications were also obtained for the involvement of a GALR1/GALR2 heteroreceptor complex in the GAL (1-15)mediated actions based on the use of the specific GALR2 antagonist M871 and icv injections of GALR1 siRNA or GALR2 siRNA producing a reduction of GALR1 or GALR2, respectively.

Importantly, 5HT1AR participates in the GAL(1-15)/FLX interactions since the 5HT1AR antagonist WAY100635 blocked the behavioral effects in the FST induced by the coadministration of GAL(1-15) and FLX. The mechanism underlying the GAL(1-15)/FLX interactions involved increases in the affinity of $5 \mathrm{HT} 1 \mathrm{AR}$ agonist binding in the DG with changes also at the transcriptional level in both $\mathrm{CA} 1$ and DG. GAL(1-15)/FLX interactions affected the binding characteristics as well as the mRNA levels of $5-\mathrm{HT} 1 \mathrm{AR}$ specifically in the dorsal hippocampus while leaving the 5-HT1A mRNA levels receptor agonist binding unaffected in the dorsal raphe.

The FST is used as a behavioral test to predict the efficacy of antidepressant treatments. The FST is an attractive behavioral screen for antidepressant drugs since it is quick to perform, reliable across laboratories, and sensitive to the effects of all of the major classes of antidepressant drugs. In this test the main indication of an antidepressant-like action of any given compound is a decrease in immobility behavior (Detke et al., 1995a; Detke et al., 1995b). The scoring of the active behaviors swimming and climbing give additional information about the mechanism of action that mediates the antidepressant-like effect. In fact, selective 5-HT reuptake inhibitors decrease immobility and increase swimming behavior (Detke et al., 1995a; Detke et al., 1995b).

In the current study FLX $10 \mathrm{mg} / \mathrm{kg}$ injected $-23,-5$ and $-1 \mathrm{~h}$ before the 5 min test significantly reduced the immobility and increased swimming time in the FST. 
These results obtained agree with previous studies and validates the behavioral model used (Estrada-Camarena et al., 2006). Moreover, FLX at 2.5mg/kg lacks effects, results which are again in agreement with those of other authors (Detke et al., 1997).

In the current work we demonstrated that GAL(1-15) enhanced FLX induced antidepressant effects in the FST. A decrease in immobility behavior and an increase of swimming behavior were observed following cotreatment with threshold doses of GAL(1-15) and of FLX (2.5 mg/kg). When administered alone, neither GAL(1-15) $1 \mathrm{nmol}$ nor $2.5 \mathrm{mg} / \mathrm{kg}$ of $\mathrm{FLX}$ affected the performance in the test, indicating that GAL(1-15) and FLX interact to provoke the enhanced antidepressant like responses. Since GAL(1-15) at an effective dose induced a pro-depressive effect (Millon et al., 2015), the antidepressant effect could only be due to the enhancement of FLX action. Moreover, the strong enhancement by GAL(1-15) of the antidepressant FLX action was validated using an effective dose of FLX.

It was demonstrated that GAL(1-15) involves GALR1-GALR2 interactions to enhance the antidepressant effects of FLX in the FST. GALR2 is involved in the GAL(1-15) effects since the GALR2 antagonist M871 blocked the enhancement of the FLX antidepressant effects induced by GAL(1-15). We were also able to block the ability of GAL(1-15) to enhance the FLX induced behavioral effects through use of rats with in vivo siRNA induced GALR1 or GALR2 knockdown, validating the concept that $\operatorname{GAL}(1-15)$ actions depend on the existence of GALR1-GALR2 heteroreceptor complexes in the brain.

Moreover in siRNA GALR1 and GALR2 knockdown rats, FLX alone reduced the immobility and increased swimming time in the FST, showing that the effect of FLX is not affected by the reduction of GALR1 or GALR2 expression.

We have previously described that GAL(1-15) alone acts through GALR1GALR2 heteroreceptor complexes which preferentially bind GAL(1-15) (Borroto-Escuela et al., 2014; Fuxe et al., 2012; Millon et al., 2016; Millon et al., 2015) and these heteroreceptor complexes were observed in the nerve cells of the dorsal hippocampus and DR (Millon et al., 2015). The results in the current paper validate that the effects of GAL(1-15) through GALR1-GALR2 
hetereceptor complexes could be a key mechanism in depression. Furthermore, the enhancing effects of GAL(1-15) on FLX mediated actions in the FST were specific, since no effects were induced by GAL, which validates and extends the view of a specific role of GAL(1-15) in brain communication.

The fact that the enhancement of the antidepressant action of FLX by GAL(115) was blocked by the 5-HT1AR antagonist WAY100635 indicates that the 5HT1AR has a significant participation in this interaction. Several authors have reported that WAY100635 when administered together with an effective dose of FLX, cancels the action of this SSRI (Estrada-Camarena et al., 2006; Serres et al., 2000). However, if the antagonist is administered in a different time point, like in the current study, the antidepressant-like activity of FLX in the FST is maintained (Estrada-Camarena et al., 2006; Serres et al., 2000). This observation of a time-schedule of injection in which WAY100635 could not prevent the antidepressant-like activity of FLX alone in the FST is of high interest. It supports the notion that GAL(1-15) through agonist activation of allosteric GALR1-GALR2-5-HT1A receptor-receptor interactions in putative trimeric heteroreceptor complexes may include effects on the recognition, pharmacology and signaling of the participating 5-HT1AR protomers. Such events can facilitate the understanding of the mechanism for the enhancement of the antidepressant actions of FLX by GAL(1-15).

We have previously described that GAL(1-15) alone acts through GALR1GALR2 isoreceptor complexes (Fuxe et al. 2012; Fuxe et al. 2008; Millon et al. 2015; Borroto-Escuela et al. 2014). These complexes could operate via allosteric GALR1-GALR2 interactions that inhibit the Gq/G11-mediated signaling of the GALR2 protomer and switches the isoreceptor complex towards Gi/o-mediated signaling (Borroto-Escuela et al. 2014; Millon et al. 2015).

The FLX induced increases in extracellular levels of 5-HT may also favor the formation of putative higher order GALR1-GALR2-5-HT1A heteroreceptor complexes. In such a complex altered allosteric receptorreceptor interactions can develop with an ability of the GALR1-GALR2 
component to enhance the 5-HT1A protomer signalling. This possibility remains to be tested.

Because not only 5-HT1AR, but also 5-HT4, 5-HT2A, 5-HT3 and 5-HT7 receptors are involved in modulating the effects of antidepressant treatments, the participation of other 5-HT receptor subtypes in the interaction should also be analized.

We have recently described that $\mathrm{GAL}(1-15)$ enhanced the antidepressant effects induced by the 5-HT1AR agonist 8-OH-DPAT in the FST (Millon et al., 2016). Thus, a significant decrease in the immobility and an increase in climbing behavior appeared after the coadministration of GAL(1-15) and 8-OH-DPAT and an increase of the swimming behavior versus the 8-OH-DPAT alone group was also observed (Millon et al., 2016). These antidepressant effects were independent of the locomotor activity, as the total distances reached and speed were equivalent between all the group (Millon et al., 2016). In the current work FLX, as a selective serotonin reuptake inhibitor binds to the serotonin transporter (SERT), thereby blocking 5-HT uptake and enhancing 5$\mathrm{HT}$ volume transmission through decreasing the clearance of extracellular 5-HT levels (Fuxe et al., 2010).

This increase in extracellular levels of $5-\mathrm{HT}$ leads to an activation of a panorama of 5-HT receptor subtypes including 5-HT1A homo and heteroreceptor complexes. We do not know which are the major types of 5-HT homo and heteroreceptor complexes involved in the antidepressant effects of FLX but the putative 5-HT1AR-GALR1-GALR2 heteroreceptor complexes are likely one relevant target for the mediation of the enhancement by $\mathrm{GAL}(1-15)$ of the antidepressant actions of FLX.

In previous work it was found that $\mathrm{GAL}(1-15)$ affected the binding characteristics as well as the mRNA levels of 5-HT1AR in the dorsal hippocampus and DR (Millon et al., 2016). In this work we observed a modification in the binding characteristics and also in the mRNA levels of 5HT1AR after coadministration of FLX and GAL(1-15). In fact, the coadministration of GAL(1-15) and FLX induced an increase in the mRNA levels of postjunctional 5-HT1AR in the dorsal hippocampus and a decrease in $\mathrm{Kd}$ and 
Bmax values, specifically in the DG of the hippocampus. These results indicate an important role of postjunctional hippocampal 5-HT1AR and their expression in the GAL(1-15)-FLX interaction. Thus, no effects were observed in the DR on the binding characteristics of soma-dendritic $5-\mathrm{HT} 1 \mathrm{~A}$ autoreceptors nor on their expression.

In previous work, we observed that 10 minutes after icv GAL(1-15) administration, no effect was observed in the 5HT-1A receptors in the DR (Millon et al., 2016). Our results suggest that in the sort term, the DR is not the key nucleus in the antidepressant effects induced by GAL(1-15). However we cannot exclude that a potential enhancement in the firing rate of the ascending 5-HT DR neurons was induced by GAL(1-15) by counteracting the 5-HT induced autoreceptor signaling in spite of lack of effects on 5-HT1A autoreceptor recognition.

In conclusion, our results indicate that icv GAL (1-15) enhances the antidepressant effects induced by FLX in the FST. The target for GAL(1-15) may mainly be 5-HT1AR-GALR1-GALR2 heteroreceptor complexes and their allosteric receptor-receptor interactions located postjunctionally in the dorsal hippocampus (Fuxe et al., 2012; Fuxe et al., 2008; Millon et al., 2016; Millon et al., 2015). In contrast, GAL appears to mainly target GALR1-5-HT1A and GALR2-5-HT1A heteroreceptor complexes (Borroto-Escuela et al., 2010; Millon et al., 2016; Millon et al., 2015). The results open up the possibility to use intranasal GAL(1-15) as a combination therapy with FLX as a novel strategy for treatment of depression.

\section{Funding and Disclosure}

This work was supported by grants awarded by Spanish Ministry of Economy (SAF2016-79008-P), PSI2013-44901-P (Grant BES-2014-068426), by the Generalitat de Catalunya (SGR2014/798) to G.M. and by UMA postdoctoral program to C.M.

The authors declare no conflict of interest. 


\section{REFERENCES}

Albert, P. R., Le Francois, B., Millar, A. M., 2011. Transcriptional dysregulation of 5HT1A autoreceptors in mental illness. Mol Brain 4, 21.

Artigas, F., 2013. Serotonin receptors involved in antidepressant effects. Pharmacol Ther 137, 119-131.

Barde, S., Ruegg, J., Prud'homme, J., Ekstrom, T. J., Palkovits, M., Turecki, G., Bagdy, G., Ihnatko, R., Theodorsson, E., Juhasz, G., Diaz-Heijtz, R., Mechawar, N., Hokfelt, T. G., 2016. Alterations in the neuropeptide galanin system in major depressive disorder involve levels of transcripts, methylation, and peptide. Proc Natl Acad Sci U S A 113, E8472-E8481.

Bartfai, T., Lu, X., Badie-Mahdavi, H., Barr, A. M., Mazarati, A., Hua, X. Y., Yaksh, T., Haberhauer, G., Ceide, S. C., Trembleau, L., Somogyi, L., Krock, L., Rebek, J., Jr., 2004. Galmic, a nonpeptide galanin receptor agonist, affects behaviors in seizure, pain, and forced-swim tests. Proc Natl Acad Sci U S A 101, 10470-10475.

Bellido, I., Diaz-Cabiale, Z., Jimenez-Vasquez, P. A., Andbjer, B., Mathe, A. A., Fuxe, K., 2002. Increased density of galanin binding sites in the dorsal raphe in a genetic rat model of depression. Neurosci Lett 317, 101-105.

Blier, P., de Montigny, C., 1994. Current advances and trends in the treatment of depression. Trends Pharmacol Sci 15, 220-226.

Borroto-Escuela, D. O., Narvaez, M., Di Palma, M., Calvo, F., Rodriguez, D., Millon, C., Carlsson, J., Agnati, L. F., Garriga, P., Diaz-Cabiale, Z., Fuxe, K., 2014. Preferential activation by galanin 1-15 fragment of the GalR1 protomer of a GalR1-GalR2 heteroreceptor complex. Biochem Biophys Res Commun 452, 347-353.

Borroto-Escuela, D. O., Narvaez, M., Marcellino, D., Parrado, C., Narvaez, J. A., Tarakanov, A. O., Agnati, L. F., Diaz-Cabiale, Z., Fuxe, K., 2010. Galanin receptor-1 modulates 5-hydroxtryptamine-1A signaling via heterodimerization. Biochem Biophys Res Commun 393, 767-772.

Branchek, T. A., Smith, K. E., Gerald, C., Walker, M. W., 2000. Galanin receptor subtypes. Trends Pharmacol Sci 21, 109-117.

Detke, M. J., Johnson, J., Lucki, I., 1997. Acute and chronic antidepressant drug treatment in the rat forced swimming test model of depression. Exp Clin Psychopharmacol 5, 107-112.

Detke, M. J., Rickels, M., Lucki, I., 1995a. Active behaviors in the rat forced swimming test differentially produced by serotonergic and noradrenergic antidepressants. Psychopharmacology (Berl) 121, 66-72.

Detke, M. J., Wieland, S., Lucki, I., 1995b. Blockade of the antidepressant-like effects of 8-OH-DPAT, buspirone and desipramine in the rat forced swim test by 5HT1A receptor antagonists. Psychopharmacology (Berl) 119, 47-54.

Diaz-Cabiale, Z., Parrado, C., Fuxe, K., Agnati, L., Narvaez, J. A., 2007. Receptorreceptor interactions in central cardiovascular regulation. Focus on neuropeptide/alpha(2)-adrenoreceptor interactions in the nucleus tractus solitarius. J Neural Transm 114, 115-125.

Diaz-Cabiale, Z., Parrado, C., Narvaez, M., Millon, C., Puigcerver, A., Fuxe, K., Narvaez, J. A., 2010. Neurochemical modulation of central cardiovascular control: the integrative role of galanin. EXS 102, 113-131. 
Diaz-Cabiale, Z., Parrado, C., Vela, C., Razani, H., Covenas, R., Fuxe, K., Narvaez, J. A., 2005. Role of galanin and galanin(1-15) on central cardiovascular control. Neuropeptides 39, 185-190.

Estrada-Camarena, E., Fernandez-Guasti, A., Lopez-Rubalcava, C., 2003. Antidepressant-like effect of different estrogenic compounds in the forced swimming test. Neuropsychopharmacology 28, 830-838.

Estrada-Camarena, E., Lopez-Rubalcava, C., Fernandez-Guasti, A., 2006. Facilitating antidepressant-like actions of estrogens are mediated by 5-HT1A and estrogen receptors in the rat forced swimming test. Psychoneuroendocrinology 31, 905914.

Fuxe, K., Borroto-Escuela, D. O., Romero-Fernandez, W., Tarakanov, A. O., Calvo, F., Garriga, P., Tena, M., Narvaez, M., Millon, C., Parrado, C., Ciruela, F., Agnati, L. F., Narvaez, J. A., Diaz-Cabiale, Z., 2012. On the existence and function of galanin receptor heteromers in the central nervous system. Front Endocrinol (Lausanne) $3,127$.

Fuxe, K., Dahlstrom, A. B., Jonsson, G., Marcellino, D., Guescini, M., Dam, M., Manger, P., Agnati, L., 2010. The discovery of central monoamine neurons gave volume transmission to the wired brain. Prog Neurobiol 90, 82-100.

Fuxe, K., Marcellino, D., Rivera, A., Diaz-Cabiale, Z., Filip, M., Gago, B., Roberts, D. C., Langel, U., Genedani, S., Ferraro, L., de la Calle, A., Narvaez, J., Tanganelli, S., Woods, A., Agnati, L. F., 2008. Receptor-receptor interactions within receptor mosaics. Impact on neuropsychopharmacology. Brain Res Rev 58, 415-452.

Fuxe, K., Ogren, S. O., Jansson, A., Cintra, A., Harfstrand, A., Agnati, L. F., 1988. Intraventricular injections of galanin reduces 5-HT metabolism in the ventral limbic cortex, the hippocampal formation and the fronto-parietal cortex of the male rat. Acta Physiol Scand 133, 579-581.

Hedlund, P. B., Fuxe, K., 1996. Galanin and 5-HT1A receptor interactions as an integrative mechanism in 5-HT neurotransmission in the brain. Ann N Y Acad Sci 780, 193-212.

Hedlund, P. B., Yanaihara, N., Fuxe, K., 1992. Evidence for specific N-terminal galanin fragment binding sites in the rat brain. Eur J Pharmacol 224, 203-205.

Jans, L. A., Riedel, W. J., Markus, C. R., Blokland, A., 2007. Serotonergic vulnerability and depression: assumptions, experimental evidence and implications. Mol Psychiatry 12, 522-543.

Jorgensen, H., Kjaer, A., Warberg, J., Knigge, U., 2001. Differential effect of serotonin $5-\mathrm{HT}(1 \mathrm{~A})$ receptor antagonists on the secretion of corticotropin and prolactin. Neuroendocrinology 73, 322-333.

Juhasz, G., Hullam, G., Eszlari, N., Gonda, X., Antal, P., Anderson, I. M., Hokfelt, T. G., Deakin, J. F., Bagdy, G., 2014. Brain galanin system genes interact with life stresses in depression-related phenotypes. Proc Natl Acad Sci U S A 111, E1666-1673.

Kehr, J., Yoshitake, T., Wang, F. H., Razani, H., Gimenez-Llort, L., Jansson, A., Yamaguchi, M., Ogren, S. O., 2002. Galanin is a potent in vivo modulator of mesencephalic serotonergic neurotransmission. Neuropsychopharmacology 27, 341-356.

Kuteeva, E., Hokfelt, T., Wardi, T., Ogren, S. O., 2008. Galanin, galanin receptor subtypes and depression-like behaviour. Cell Mol Life Sci 65, 1854-1863.

Lu, X., Barr, A. M., Kinney, J. W., Sanna, P., Conti, B., Behrens, M. M., Bartfai, T., 2005. A role for galanin in antidepressant actions with a focus on the dorsal raphe nucleus. Proc Natl Acad Sci U S A 102, 874-879. 
Millon, C., Flores-Burgess, A., Narvaez, M., Borroto-Escuela, D. O., Santin, L., Gago, B., Narvaez, J. A., Fuxe, K., Diaz-Cabiale, Z., 2016. Galanin (1-15) enhances the antidepressant effects of the 5-HT1A receptor agonist 8-OH-DPAT: involvement of the raphe-hippocampal 5-HT neuron system. Brain Struct Funct.

Millon, C., Flores-Burgess, A., Narvaez, M., Borroto-Escuela, D. O., Santin, L., Parrado, C., Narvaez, J. A., Fuxe, K., Diaz-Cabiale, Z., 2015. A role for galanin Nterminal fragment (1-15) in anxiety- and depression-related behaviors in rats. Int J Neuropsychopharmacol 18.

Misane, I., Razani, H., Wang, F. H., Jansson, A., Fuxe, K., Ogren, S. O., 1998. Intraventricular galanin modulates a 5-HT1A receptor-mediated behavioural response in the rat. Eur J Neurosci 10, 1230-1240.

Mitsukawa, K., Lu, X., Bartfai, T., 2008. Galanin, galanin receptors and drug targets. Cell Mol Life Sci 65, 1796-1805.

Pompeiano, M., Palacios, J. M., Mengod, G., 1992. Distribution and cellular localization of mRNA coding for 5-HT1A receptor in the rat brain: correlation with receptor binding. J Neurosci 12, 440-453.

Razani, H., Diaz-Cabiale, Z., Misane, I., Wang, F. H., Fuxe, K., Ogren, S. O., 2001. Prolonged effects of intraventricular galanin on a 5-hydroxytryptamine(1A) receptor mediated function in the rat. Neurosci Lett 299, 145-149.

Saenz del Burgo, L., Cortes, R., Mengod, G., Montana, M., Garcia del Cano, G., Salles, J., 2013. Chronic effects of corticosterone on GIRK1-3 subunits and 5-HT1A receptor expression in rat brain and their reversal by concurrent fluoxetine treatment. Eur Neuropsychopharmacol 23, 229-239.

Serres, F., Muma, N. A., Raap, D. K., Garcia, F., Battaglia, G., Van de Kar, L. D., 2000. Coadministration of 5-hydroxytryptamine(1A) antagonist WAY-100635 prevents fluoxetine-induced desensitization of postsynaptic 5-hydroxytryptamine(1A) receptors in hypothalamus. J Pharmacol Exp Ther 294, 296-301.

Tatemoto, K., Rokaeus, A., Jornvall, H., McDonald, T. J., Mutt, V., 1983. Galanin - a novel biologically active peptide from porcine intestine. FEBS Lett 164, 124-128.

Tomiyama, M., Palacios, J. M., Cortes, R., Vilaro, M. T., Mengod, G., 1997. Distribution of AMPA receptor subunit mRNAs in the human basal ganglia: an in situ hybridization study. Brain Res Mol Brain Res 46, 281-289.

Wang, P., Li, H., Barde, S., Zhang, M. D., Sun, J., Wang, T., Zhang, P., Luo, H., Wang, Y., Yang, Y., Wang, C., Svenningsson, P., Theodorsson, E., Hokfelt, T. G., Xu, Z. Q., 2016. Depression-like behavior in rat: Involvement of galanin receptor subtype 1 in the ventral periaqueductal gray. Proc Natl Acad Sci U S A 113, E4726-4735.

Wang, S., He, C., Maguire, M. T., Clemmons, A. L., Burrier, R. E., Guzzi, M. F., Strader, C. D., Parker, E. M., Bayne, M. L., 1997. Genomic organization and functional characterization of the mouse GalR1 galanin receptor. FEBS Lett 411, 225-230.

Weiss, J. M., Bonsall, R. W., Demetrikopoulos, M. K., Emery, M. S., West, C. H., 1998. Galanin: a significant role in depression? Ann N Y Acad Sci 863, 364-382.

\section{Figure legends}

Figure 1. Behavioural effects of the coadministration of effective dose of FLX $(10 \mathrm{mg} / \mathrm{Kg})$ and $\mathrm{GAL}(1-15)(1 \mathrm{nmol})$ or Galanin $(1 \mathrm{nmol})$ in the FST. FLX was administered subcutaneously (sc) 23, 5 and 1 hour before the test. CSFa, 
GAL(1-15) and Galanin were injected intracerebroventricular (icv) 15 min before the test. Data represents mean \pm SEM of immobility, climbing and swimming time in FST during the 5 min test period ( $n=7-8$ rats per group). ${ }^{*} \mathrm{p}<0.05$, ${ }^{* *} p<0.01$ versus all the groups; $\# p<0.05$ versus Control according to one way ANOVA followed by Newman-Keuls Multiple Comparison Test.

Figure 2. Behavioural effects of the coadministration of effective dose of FLX $(10 \mathrm{mg} / \mathrm{Kg})$ and threshold doses of GAL(1-15) (1nmol) in (A) Sprague-Dawley rats which also were treated with GALR2 antagonist M871 (3nmol) or (B) GALR2 knockdown rats (siRNA GALR2). FLX or vehicle was administered subcutaneously (sc) 23, 5 and 1 hour before the test. aCSF, GAL(1-15) and GAL(1-15)+M871 were injected intracerebroventricularly (icv) 15 min before the test. Data represents mean \pm SEM of immobility, climbing and swimming times in FST during the 5 min test period ( $n=7-9$ rats per group). $\# p<0.05$, \#\#p<0.01 versus FLX10mg(sc)+CSFa(icv) and FLX10mg(sc)+GAL(1-15) 1nmol+M871 $3 \mathrm{nmol}$ (icv); ${ }^{*} \mathrm{p}<0.05,{ }^{* *} \mathrm{p}<0.01$ versus Control according to one way ANOVA followed by Newman-Keuls Multiple Comparison Test.

Figure 3. Behavioural effects of effective dose of $F L X \quad 10 \mathrm{mg} / \mathrm{Kg}(\mathbf{A})$ and coadministration of FLX $10 \mathrm{mg} / \mathrm{Kg}$ and threshold doses of $\mathrm{GAL}(1-15)(1 \mathrm{nmol})(B)$ in GALR1 knockdown rats (siGalR1). FLX and vehicle was administered sc 23, 5 and 1 hour before the test. CSFa, GAL(1-15) were injected icv 15 min before the test. Data represents mean \pm SEM of immobility, climbing and swimming time in FST during the 5 min test period ( $n=7-9$ rats per group) ${ }^{*} p<0.05$ according to Student's unpaired t-test. (C) GALR1 protein expression eight days after a single i.c.v. injection of siRNA GALR1 or vehicle into the rat brain. Data are represents as mean $\pm S E M$ of the percentages respect the control value $(100 \%)$ of optic density (O.D) ${ }^{*} p<0.05$ according to Student's unpaired t-test. (D) Representative photographs of GALR1 immunohistochemistry

Figure 4. Behavioural effects of the coadministration of effective dose of FLX $(10 \mathrm{mg} / \mathrm{Kg})$ and $5-\mathrm{HT} 1 \mathrm{~A}$ antagonist WAY100635 (6nmol) and the coadministration of effective dose of FLX $(10 \mathrm{mg} / \mathrm{Kg})$ with $\mathrm{GAL}(1-15)(1 \mathrm{nmol})$ 
and WAY100635 (6nmol) in the FST. FLX was administered sc 23, 5 and 1 hour before the test. GAL(1-15), WAY100635 and GAL(1-15)+WAY100635 were injected icv 15 min before the test. Data represents mean \pm SEM of immobility, climbing and swimming times in FST during the 5 min test period ( $n=5-10$ rats per group). ${ }^{*} p<0.05,{ }^{* *} p<0.01$ versus all groups according to one-way ANOVA followed by Newman-Keuls Multiple Comparison Test.

Figure 5. (A) Effects of the coadministration of FLX (10mg/Kg) and GAL(1-15) (1nmol) on 5-HT1AR mRNA levels in dorsal hippocampus (CA1 and DG). FLX was administered sc 23, 5 and 1,25 hour before sacrifice and GAL(1-15) was injected icv 30 min before sacrifice. The values of optical density (O.D.), mean \pm SEM ( $n=6$ per group), are expressed as percentages respect of the control values $(100 \%)$. ${ }^{*} p<0.05$ versus all groups according to one way ANOVA followed by Newman-Keuls Multiple Comparison Test (B) Representative autoradiograms from dorsal hippocampus showing the levels of mRNA coding for 5-HT1AR determined by in situ hybridization and the analyzed areas.

Figure 6. Effects on the binding characteristics of 5-HT1AR agonist $[3 \mathrm{H}]-8-\mathrm{OH}-$ DPAT in the dental gyrus (DG) of the hippocampus (A) after the coadministration of FLX $(10 \mathrm{mg} / \mathrm{Kg})$ and aCSF or GAL(1-15) (1nmol). FLX was administered sc 23, 5 and 1,25 hour before sacrifice and GAL(1-15) or CSFa was injected icv $30 \mathrm{~min}$ before. Saturation experiments were performed with ten concentrations of $\left[{ }^{3} \mathrm{H}\right]-8-\mathrm{OH}-\mathrm{DPAT}(0.26-10 \mathrm{nM})$ in sections from the Hippocampus. Non-specific binding was defined as the binding in the presence of $10 \mu \mathrm{M}$ serotonin. The $\mathrm{Kd}$ and the Bmax values are shown as mean \pm SEM ( $\mathrm{n}=6$ per group). In $\mathrm{FLX}(10 \mathrm{mg} / \mathrm{kg})$ the $\mathrm{Kd}(\mathrm{nM})$ value was $1.2 \pm 0.1$ and $B \max$ (fmol/mg prot) was $545 \pm 37$. ${ }^{*} \mathrm{p}<0.05 ;{ }^{* *} \mathrm{p}<0.01$ according to Student's unpaired t-test. (B) Representative autoradiograms from Hippocampus sections of rat showing an increase of the afinity of the agonist for $5-\mathrm{HT} 1 \mathrm{AR}$ with a low concentration of the radioligand $(1 \mathrm{nM})$. 
Figure 1.

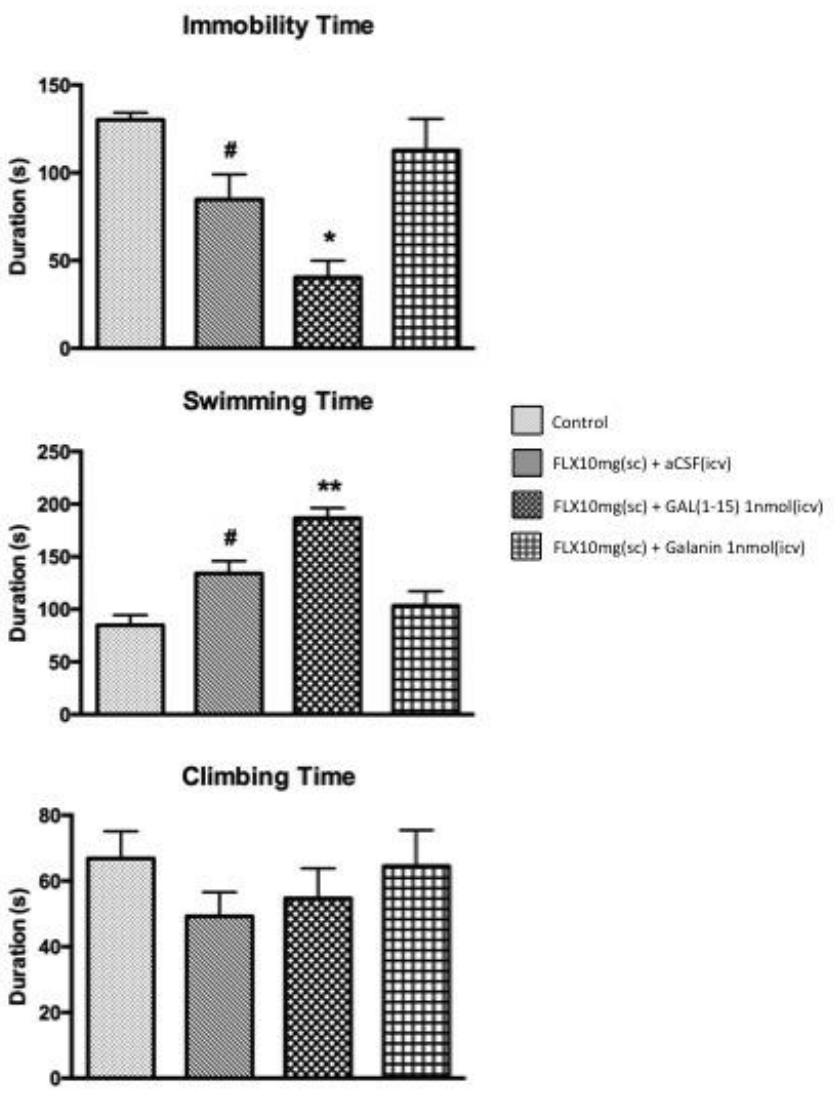

Figure 2
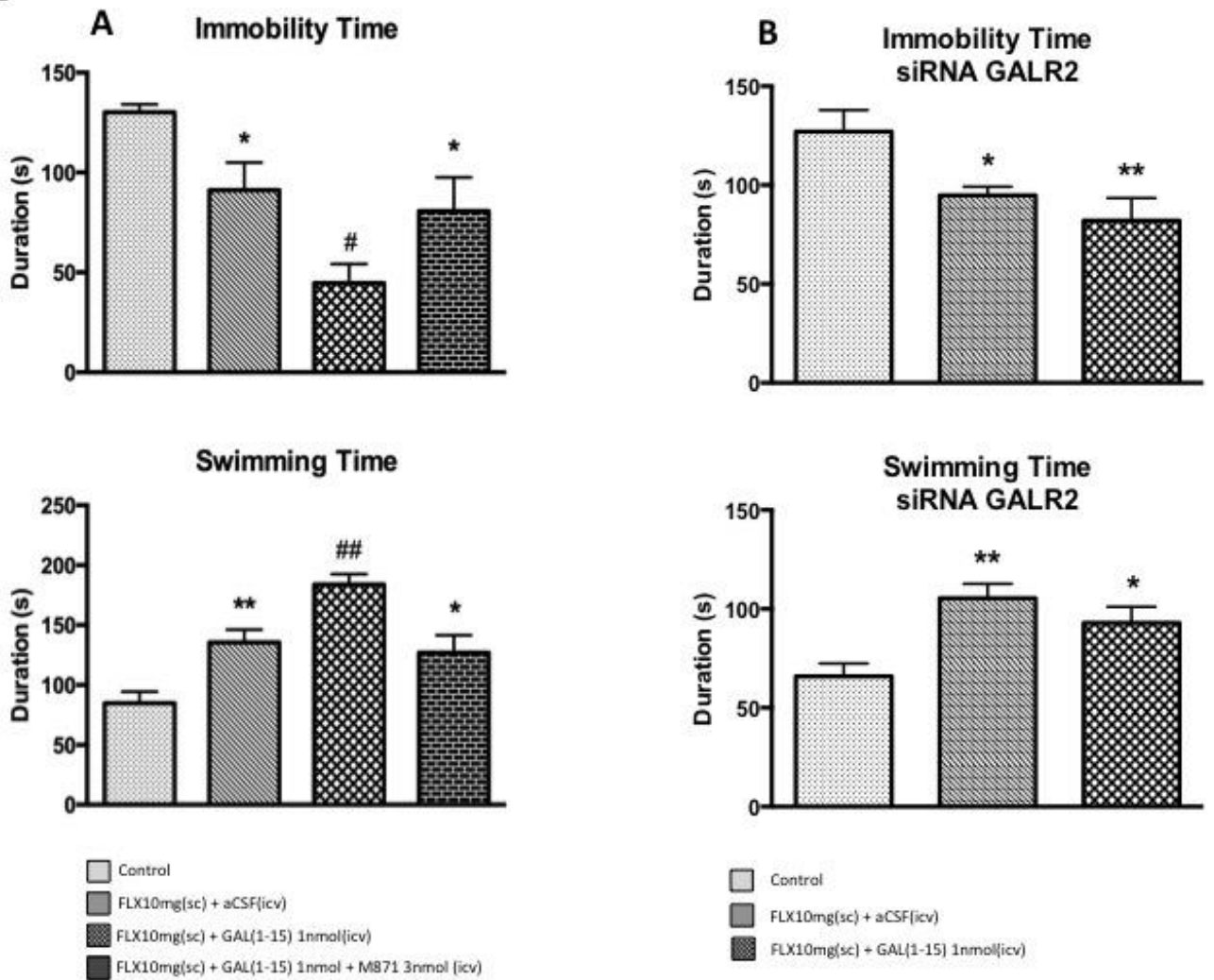
Figure 3

A

\begin{tabular}{cccc}
\hline & $\begin{array}{c}\text { Immobility } \\
\text { Time } \\
\text { (siRnA GALR1) }\end{array}$ & $\begin{array}{c}\text { Swimming } \\
\text { Time } \\
\text { (siRNA GALR1) }\end{array}$ & $\begin{array}{c}\text { Climbing } \\
\text { Time } \\
\text { (siRNA GALR1) }\end{array}$ \\
\hline Control & $101,5 \pm 7$ & $88,8 \pm 8$ & $93,2 \pm 11$ \\
FLX (10 & $\left.70,9 \pm 8^{*} / \mathrm{Kg}\right)$ & $107,8 \pm 5,8^{*}$ & $92,3 \pm 14$ \\
\hline
\end{tabular}

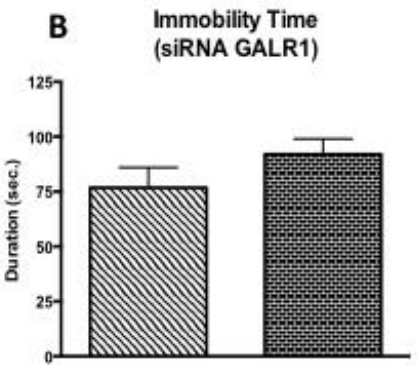

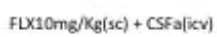

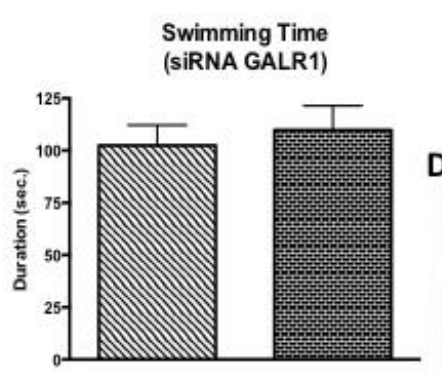

FLX10mg/Kg(sc) + GALL1-15)(1nmal)(ivv]

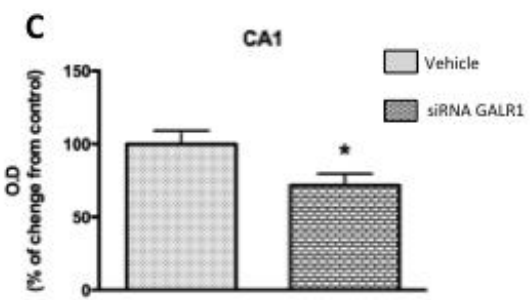

DG

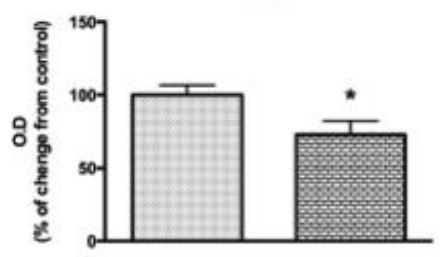

D

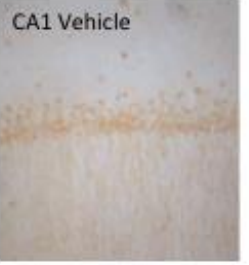

CA1 SIRNA GALR1

Figure 4

Immobility Time

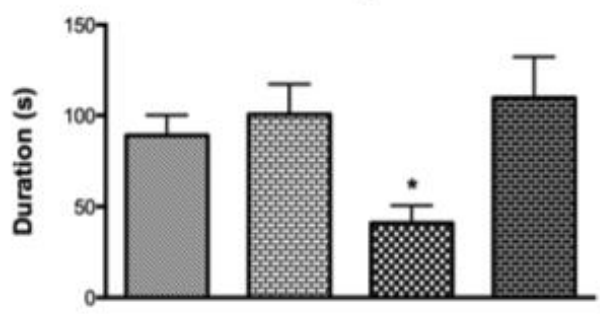

$\square$ FLX10mglsc) + aCSF(icv'

FLX10mg(sc) + WAY100635 $6 \mathrm{nmol}(\mathrm{icv})$

FLX10mg(sc) + GAL[1-15) 1nmol(iv)]

Swimming Time

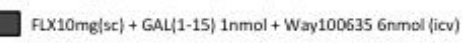

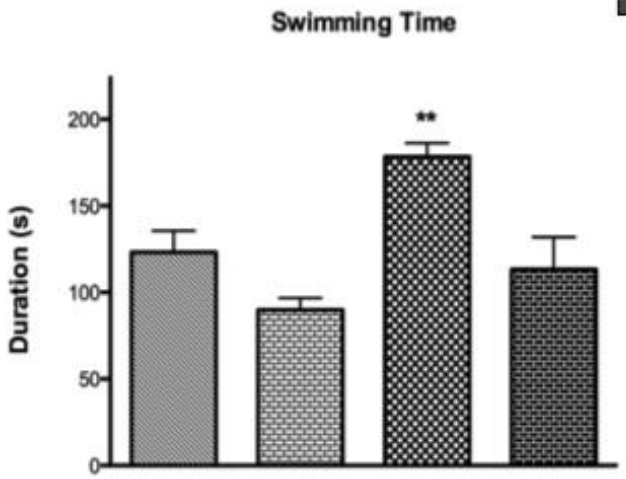


A

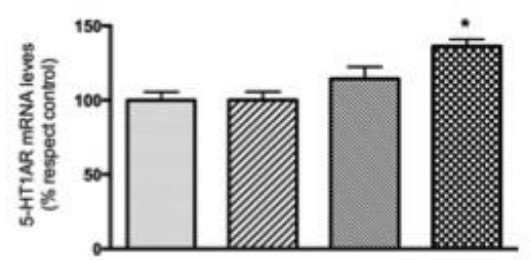

DG

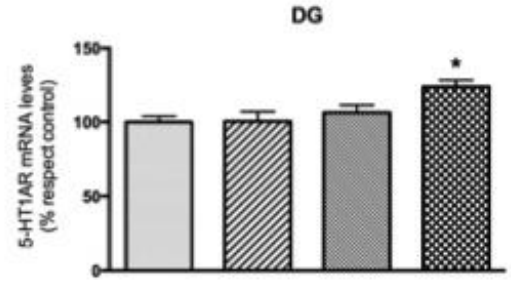

B
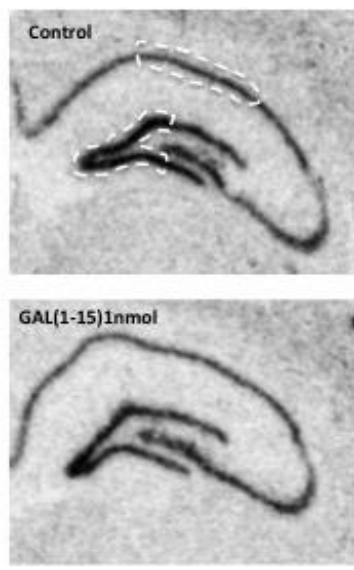

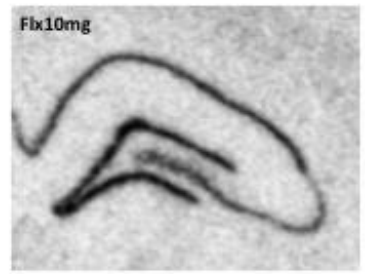

Flx10mg + GAL(1-5)1nmo

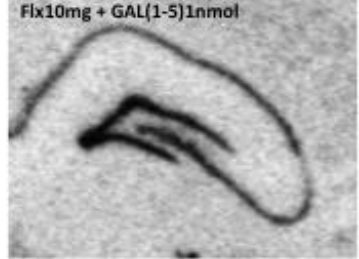

Fig.5

A

Kd - DG
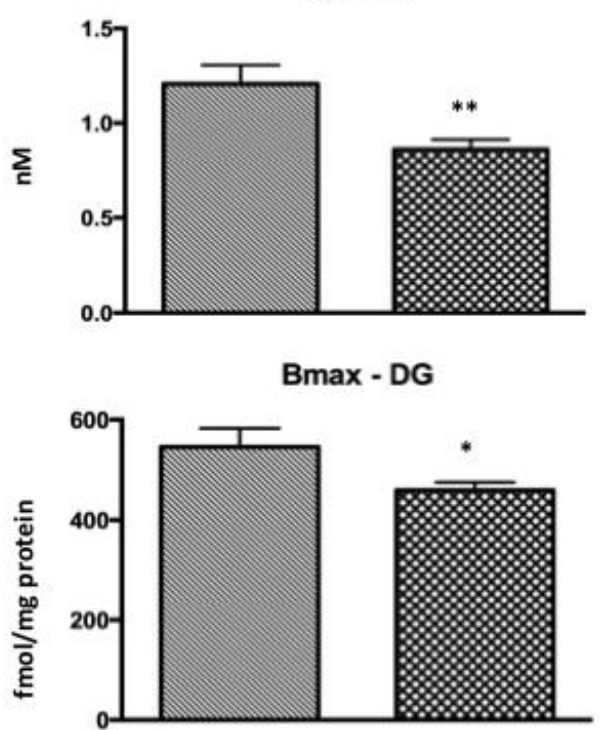

B
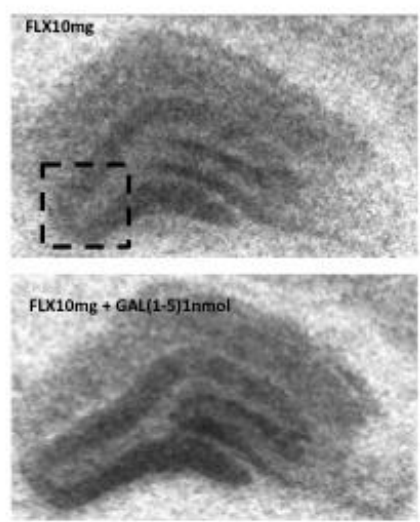

Flx10mg|sc) + CSFa(ivv)

聯 Flx10mg(sc) + GAL(1-15) 1nmollicv

Fig. 6 